\title{
An efficient NC tool path planning approach
}

\author{
K.S. TSUI \\ Department of Mechanical Engineering \\ The University of Hong Kong, H.K. \\ kstsui@graduate.hku.hk
}

\author{
K.W. CHAN \\ Department of Mechanical Engineering, \\ The University of Hong Kong, H.K. \\ akwchan@hkucc.hku.hk
}

\begin{abstract}
One major problem encountered in NC machining is that cutting load fluctuates in twisty tool paths and increases momentarily when milling concave corners where stock material concentrates. Much research has been conducted on controlling milling forces but the problem remains unsolved. This paper presents a solution to reduce and stabilise cutting load in NC milling operation by applying computational techniques to isolate the concave corners of a machining region, thereby forming a major region and a number of disjoint sub-regions. Due to the suppression of concave corners, the boundary profile of the major region will become smoother. Contour-parallel offset tool paths can then be used to remove the material in the major region. For the isolated sub-regions, special corner removing tool paths with the use of different cutter sizes will be employed. By using this approach, the adverse effects caused by excessive undulation of milling tool path can be reduced.
\end{abstract}

\section{Introduction}

Mould and die making industry, like any other businesses, is facing ever increasing stringent customer demands such as extremely short delivery time and superior quality. A major production operation in the industry is machining, which contribute to more than $50 \%$ of the total mould manufacturing lead time [1] and up to $30 \%$ of the total mould making costs [2]. As a result, the industry is finding ways to reduce the production lead time and improve the quality of their products.

Reducing and stabilising cutting load, especially in High Speed Machining (HSM) of hard die steels, can produce significant benefits such as longer cutter life and shorter cutting time. Effective HSM combines higher spindle speeds and feed rates with optimized tool-paths to obtain faster manufacturing times and improved surface finishes. Consequently, time-consuming finishing operations can be reduced or eliminated.

In this paper, a practical machining strategy to reduce and stablise the cutting load in NC milling operation is presented. Section 2 reviews related work on controlling the milling forces. Section 3 describes the recommended tool path generation strategy and implementations. Section 4 discusses the feasibility of the proposed approach and proposes further work to be pursued.

\section{Background and Related Work}

Contour-parallel offset (CPO) is one of the commonly used tool paths. Since CPO tool path is derived from the sectional boundaries of a machining part, it inevitably contains convex and concave corners. The cutter engage angle encountered in a concave circular tool path is always higher than those encountered in convex and linear tool paths. This can be illustrated in Figure 1.

(Insert Figure 1. here)

The cutter engage angle can also be represented mathematically [3]: 


$$
\begin{aligned}
& \text { Linear tool path }: \cos (\alpha)=1-\frac{s}{r} \\
& \text { Concave circular tool path : } \cos (\alpha)=1-\frac{s}{r}-\frac{s(r-0.5 s)}{R r} \\
& \text { Convex circular tool path : } \cos (\alpha)=1-\frac{s}{r}+\frac{s(r-0.5 s)}{R r}
\end{aligned}
$$

where $\alpha, s, r$ and $R$ are the cutter engage angle, radial depth of cut, cutter radius and circular path radius respectively. Since the larger the engage angle, the higher the cutter

load, hence cutter load rises when cutting concave corners. The abrupt fluctuation of cutter loads leads to machine vibration, wavy finished surface and even tool breakage.

Some previous work has been done on controlling the milling forces. For instance, Wang [4] and Altinas et al [5] employed solid modeling technology to model the cutterworkpiece interaction for optimizing cutting parameters. However, the modeled results only indicate the average conditions, which may be far from instantaneous conditions.

Some researchers tried to adaptively adjust the cutting parameters instantaneously during actual cutting to satisfy the changing machining conditions. Smith et al [6] presented an algorithm for automatically selecting the optimum spindle speed based on the cutting force signal. Davies et al [7] employed capacitance probes to measure the deflection of end mills during high speed milling and compared the measured results with the predictions of regenerative chatter theory. Jerard et al [8] demonstrated the integration of geometric and mechanistic models of milling into a tool controller for improving machining productivity. But these approaches require sophisticated hardware implementation and leads to a lower machining efficiency. Tounsi et al [9] optimizes the use of a dynamic feed rate according to the changes in the cutting geometry while maintaining a near-constant cutting force. However, possible conflicts may occur between tool path geometry and the physical limits of the feed drive system.

Much research has been conducted to minimize chip load by modifying tool path trajectory. This is an efficacious approach because tool movement affects cutting load. Iwabe et al [10] attempted to reduce the chip load at cutting right-angled corners by using a looped cutter path, which removed the stock material progressively in several passes. Tsai et al [11] attempted to enhance Iwabe's corner-looping tool path but the improved tool paths still could not deal with complicated corner shapes. Hansen [12] presented an offsetting algorithm for generating contour-parallel and zig-zag tool paths for arbitrarily shaped pockets with islands. Since only geometry was considered, the generated tool paths consist of many sharp corners and idle positioning motions.

While the high chip load fluctuation problem remains unsolved, a commonly practised remedy in industry is adding round fillets to sharp changing tool path segments. This is undesirable because introducing round corners consequently creates more concave corners, and hence the problem remains. Practitioners resort on slowing down the cutter motion at sharp corners, however frequent acceleration and deceleration of cutter motion deter the use of HSM. In view of these drawbacks, a solution to reduce and stablise cutting load in NC milling operation is described in the sequel.

\section{Method and Implementation}

For a given 2D machining contour, our proposed method begins by constructing a convex hull [13] to encapsulate the contour. Effectively, the space outside the convex hull becomes a major machining region while the spaces between the convex hull and the original machining contour produce several disjoint concave corners.

(Insert Figure 2. here) 
As the convex hull boundary does not contain concave corners, CPO tool paths are generated to remove the material outside the major region. For the remaining isolated sub-regions which represent the original concave corner regions, special tool paths are proposed to remove the corner material progressively and with the use of different cutter sizes. By using this tool path planning approach, material in the major region can be machined at higher feed rates because the CPO tool paths derived from the convex hull boundary do not contain concave segments. Moreover, the use of the special corner clearing tool paths can reduce the adverse effects caused by excessive undulation of tool path in concave regions. An experimental computer program is implemented in $\mathrm{C}++$ with API routines from Unigraphics ${ }^{\circledR}$ CAD/CAM System for concept proofing. A case study has been conducted with a test piece as shown in Figure 2.

A simplified Convex Hull Generation algorithm based on Jarvis's March Algorithm [13] is implemented as follows:

(Insert Figure 3. here)

Output data: A set of $\mathrm{n}$ vertices $\left\{\mathrm{P}_{0}, \mathrm{P}_{1}, \mathrm{P}_{2}, \ldots, \mathrm{P}_{\mathrm{n}}\right\}$ of the convex hull.

Input data: Vertices from a sectional boundary. For arc segments, vertices are extracted along an arc in constant angular interval. End points are extracted from line segments.

Initialisation: $\mathrm{P}_{0}$ is the right most point, i.e. the point with the greatest $\mathrm{X}$ co-ordinate is the first vertex of convex hull.

Recursion: To find $\mathrm{P}_{\mathrm{i}}$, calculate the angles between points that are not the vertices of a convex hull and the previous vertex of a convex hull. $\mathrm{P}_{\mathrm{i}}$ is found such that the angle between $\mathrm{P}_{\mathrm{i}}$ and $\mathrm{P}_{\mathrm{i}-1}$ is the smallest.

Return Condition: The algorithm runs until $\mathrm{P}_{\mathrm{i}}=\mathrm{P}_{\mathrm{o}}$. The algorithm returns when the next vertex found is the first vertex.

After all the vertices of a convex hull are found, a complete convex hull is generated by joining up the points by straight lines. Any line segment that does not belong to the original boundaries is classified as an engage edge. Areas enclosed by the engage edges and original boundaries are defined as corner machining regions, where Uni-directional linear (UDL) tool paths are employed to remove the corner materials. UDL tool path consists of a straight line tool path with arcs appended at both ends. A constant cutting load is achieved throughout the linear portion, allowing the use of higher feed rate. The approach and retract arcs are added to reduce fluctuation in cutting load when the cutter enters and leaves the material.

(Insert Figure 4. here)

Tool path generated by the proposed strategy is free of concave arc segments and is much smoother than the traditional CPO tool path as can be seen by comparing Figures 5 and 6.

(Insert Figure 5. and 6. here)

Remaining uncut stock will be formed at corner regions when the cutter radius exceeds the corner radius like a case illustrated in Figure 7. Re-roughing with the use of smaller cutter is necessary for clearing any remaining stock which can not be reached by a previous tool (Figure 8.).

(Insert Figure 7. and 8. here)

Re-roughing is done after an initial roughing has been completed. A new engage edge is subtended in the corner regions to define a new re-cutting region, where UDL tool paths will be created (Figure 9. and 10.). It reduces the subsequent slow finishing operations while achieving a higher accuracy in the machined parts.

(Insert Figure 9. and 10. here) 


\section{Discussions \& Further Works}

The proposed tool path planning approach addresses the problem of high chip load fluctuation for protrusion type features. Due to the absence of concave corners, higher material removal rate can be used. This favors the application of HSM.

Re-roughing is necessary to deal with the uneven distribution of material in the major region and in the concave corner regions. Due to the use of the convex hull boundary, higher material removal rate can be achieved in initial roughing using a relatively large cutter with a greater depth of cut. Re-roughing is then employed to remove localised remaining material at sharp concave corner regions. Without re-roughing, a finishing tool will encounter excessively large volume of material especially when reaching the concave corners. This is instanced clearly in Figure 7 that a considerable amount of material is left in a narrow slot after initial roughing. To avoid cutter overload, the current practice in industry is to use a shallower depth of cut and slower feed rate. Consequently, this leads to an inefficient machining operation.

In our present work, only UDL tool path generation is implemented for removing cornermaterial. More tool path strategies will be studied and implemented, namely the trochoidal [14] tool path and the corner-looping tool path. Trochoidal tool path is a new tool path strategy to increase rough machining speed. It is defined as a trace of a point fixed on a circle that rolls along a line. Corner-looping tool path is a variant of trochoidal tool path which removes the corner material progressively by several looping passes. The virtue of these tool paths permits the use of a more constant cutting load and feed rate. Experiments will be conducted to verify these advantages.

\section{Acknowledgment}

The authors would like to thank The Research Grants Council of Hong Kong for providing funding support (RGC Reference: HKU 7182/03E) to the work described in this paper. The provision of computing and NC machining facilities by the Department of Mechanical Engineering of The University of Hong Kong is also deeply appreciated.

\section{References}

[1] P. Fallbohmer, C.A. Rodriguez, T. Ozel, and T. Altan, "High-speed machining of cast iron and alloy steels for die and mold manufacturing", Journal of Materials Processing Technology, Elsevier Science Ltd, Jan 2000, pp. 104-115.

[2] A. Taylan, L. Blaine, and Y.C. Yen, "Manufacturing of dies and molds", CIRP Annals - Manufacturing Technology, Hallwag Publishing Ltd, USA, 2001, pp. 405-423.

[3] T.R. Kramer, "Pocket milling with tool engagement detection", Journal of Manufacturing Systems, Vol. 11(2), 1992, pp. 114-123.

[4] W.P. Wang, "Solid modeling for optimizing metal removal of three-dimensional NC end milling”, Journal of Manufacturing Systems, USA, 1998, pp. 57-65.

[5] Y. Altinas, and A. Spence, "End milling force algorithms for CAD systems", Annals of the CIRP, Int Inst for Production Engineering Research, USA, 1991, pp. 31-34.

[6] S. Smith, and J. Tlusty, "Update on high-speed milling dynamics", Journals of Eng. fro Industry, USA, 1990, pp. 142-149.

[7] M.A. Davies, B. Duttere, J.R. Pratt, and A.J. Schaut, "On the dynamics of high-speed milling with long, slender endmills”, CIRP Annals - Manufacturing Technology, Hallwag Publishing Ltd, 1998, pp. 55-60.

[8] R. Jerard, B.K. Fussell, M.T. Ercan, and J.G. Hemmett, "Integration of geometric and mechanistic models of NC machining into an open-architecture machine tool controller", Proceedings of IMECE, 2000, pp. 1-8.

[9] N. Tounsi, and M.A. Elbestawi, "Optimized feed scheduling in three axes machining. Part I: Fundamentals of the optimized feed scheduling strategy", International Journal of Machine Tools \& Manufacture, Elsevier Science Ltd, 2003, pp. 253-267. 
[10] H. Iwabe, Y.K. Fujii, K. Saito, and T. Kishinami, "Study on corner cut by end mill-Analysis of cutting mechanism and new cutting method at inside corner", Journal of Japan Soc. of Precision Eng., 1989, pp. 841-846.

[11] M.D. Tsai, S. Takata, M. Inui, F. Kimura, and T. Sata, "Operation planning based on cutting process models", Annals of CIRP, Int Inst for Production Engineering Research, USA, 1991, pp. 95 - 98.

[12] A. Hansen, and F. Arbab, “An algorithm for generating NC tool paths for arbitrarily shaped pockets with island", ACM Transactions on Graphics, Vol. 11(2), 1992, pp. 152-182.

[13] R.A. Jarvis, "On the identification of the convex hull of a finite set in the plane", Information Processing Letters, 1973, pp. 18-21.

[14] I. Yamaji, S. Ibaraki, Y. Kakino, and S. Nishida, "Tool path planning using trochoid cycles for hardened steel in die and mold manufacturing (1st Report)", Proceedings of the International Conference on Agile Manufacturing, Advances in Agile Manufacturing, China Machine Press, China, 2003, pp. 435-442.

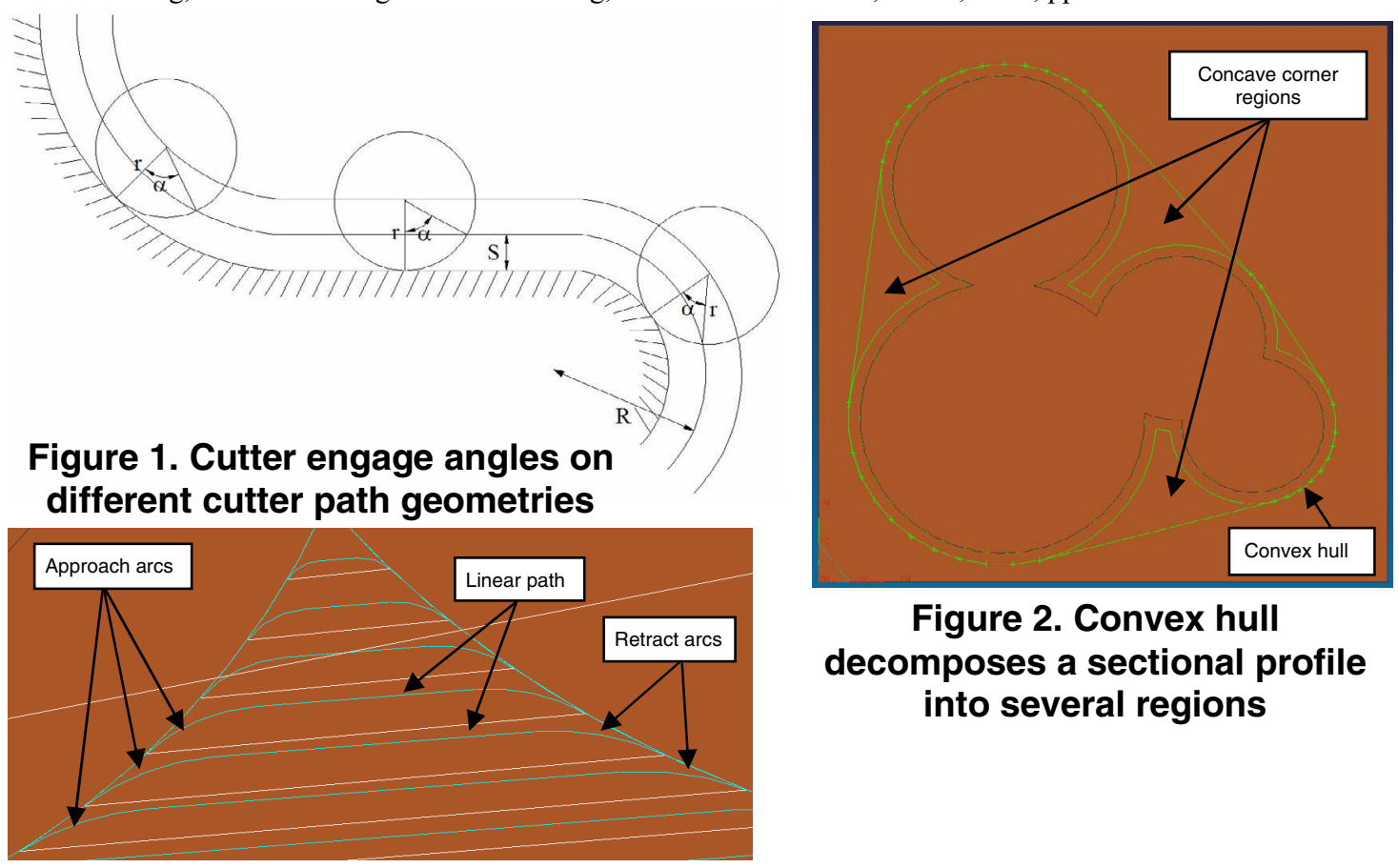

Figure 4. UDL tool path

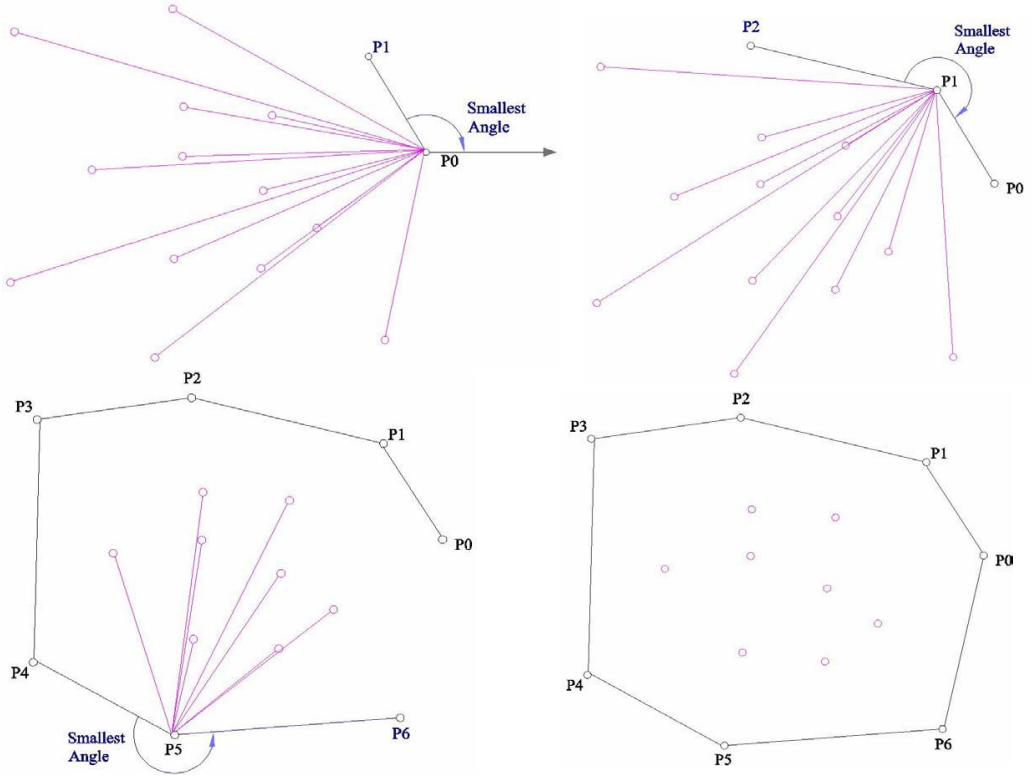

Figure 3. Jarvis's March Algorithm 

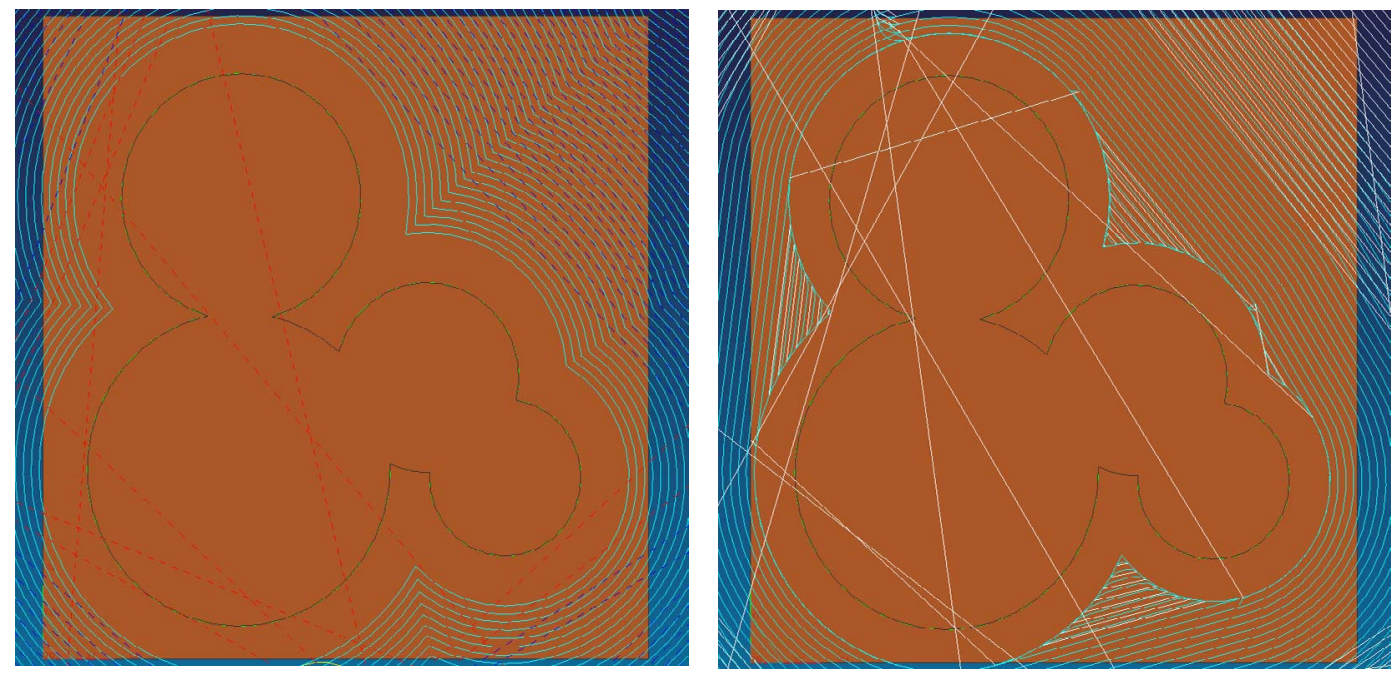

Figure 5. Conventional CPO tool path derived directly from part boundary

Figure 6. Smoother tool path

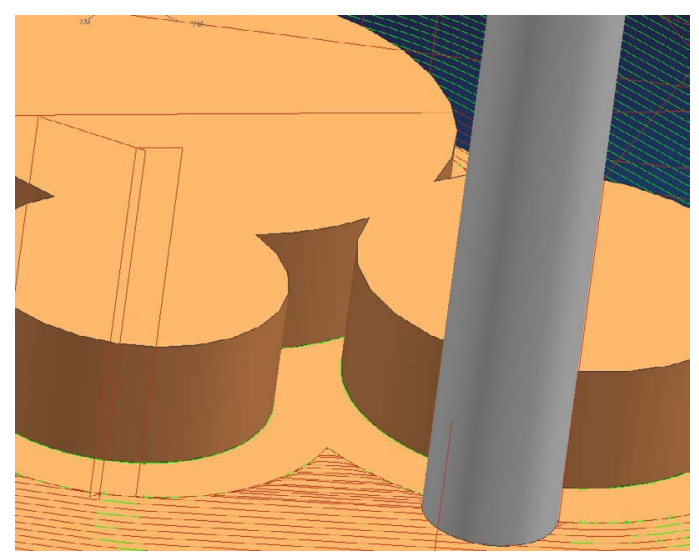
generated by the proposed strategy

Figure 7. Big cutter cannot a reach sharp corner

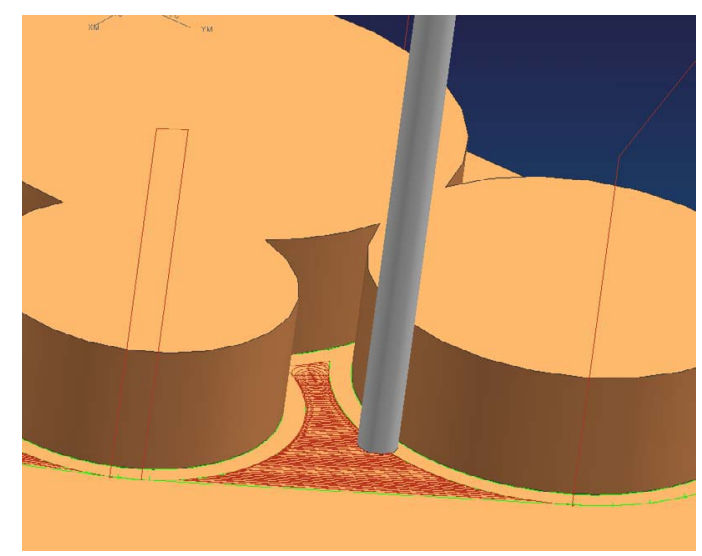

Figure 8. Re-roughing with smaller cutter

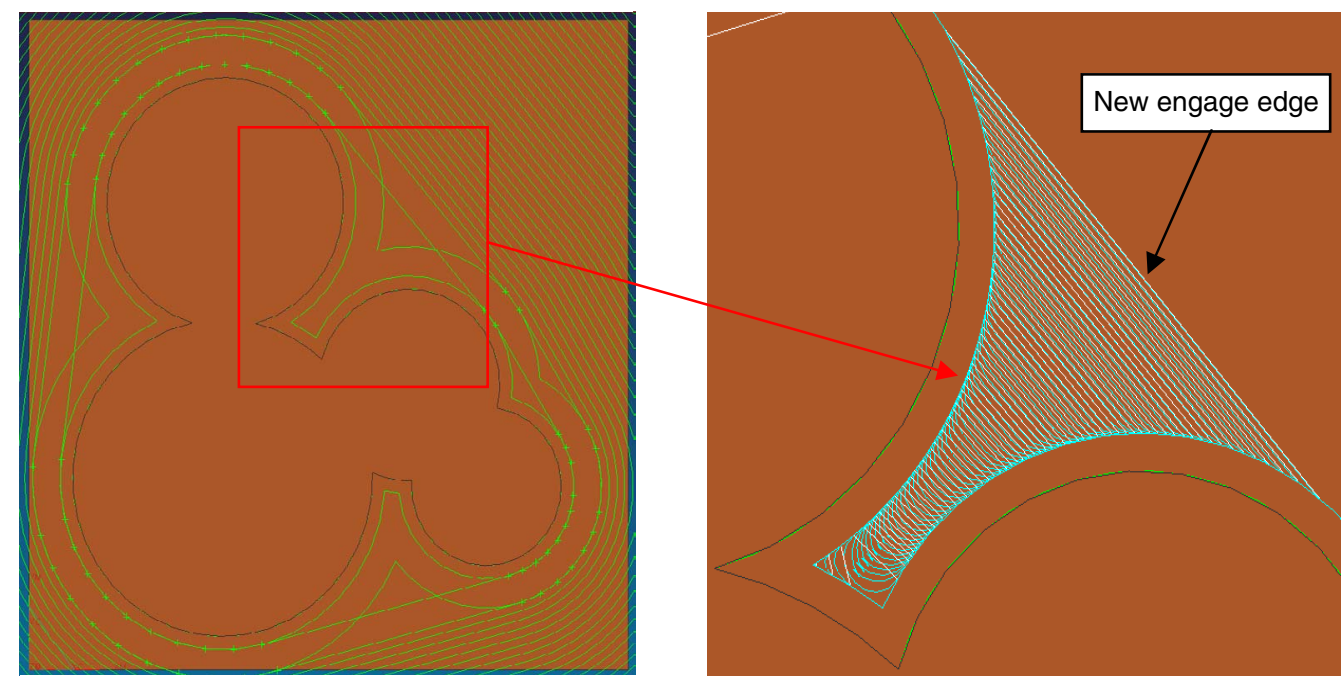

Figure 9. New machining regions formed for re-roughing

Figure 10. Re-roughing tool path to clear remaining material 